University of Wollongong

Research Online

Australian Institute for Innovative Materials -

Papers

Australian Institute for Innovative Materials

$1-1-2016$

Processable 2D materials beyond graphene: MoS2 liquid crystals and fibres

Rouhollah Jalili

University of Wollongong, rjalili@uow.edu.au

Sima Aminorroaya-Yamini

University of Wollongong, sima@uow.edu.au

Tania Benedetti Goncales

University of Wollongong, taniaben@uow.edu.au

Seyed Hamed Aboutalebi

Institute For Research In Fundamental Sciences, sha942@uowmail.edu.au

Yunfeng Chao

University of Wollongong, yc682@uowmail.edu.au

See next page for additional authors

Follow this and additional works at: https://ro.uow.edu.au/aiimpapers

Part of the Engineering Commons, and the Physical Sciences and Mathematics Commons

Research Online is the open access institutional repository for the University of Wollongong. For further information contact the UOW Library: research-pubs@uow.edu.au 


\title{
Processable 2D materials beyond graphene: MoS2 liquid crystals and fibres
}

\author{
Abstract \\ Herein, we show properly engineered MoS2 crystals can readily form liquid crystalline dispersions in \\ water making them ideal candidates for large-scale manufacturing processes. The guideline provided \\ here can serve as the basis to develop practical protocols to address the long-standing goal of large-scale \\ manufacturing of 2D materials. \\ Disciplines \\ Engineering | Physical Sciences and Mathematics \\ Publication Details \\ Jalili, R., Aminorroaya-Yamini, S., Benedetti, T. M., Aboutalebi, S., Chao, Y., Wallace, G. G. \& Officer, D. L. \\ (2016). Processable 2D materials beyond graphene: MoS2 liquid crystals and fibres. Nanoscale, 8 (38), \\ 16862-16867. \\ Authors \\ Rouhollah Jalili, Sima Aminorroaya-Yamini, Tania Benedetti Goncales, Seyed Hamed Aboutalebi, Yunfeng \\ Chao, Gordon G. Wallace, and David L. Officer
}




\section{Journal Name}

\section{COMMUNICATION}

\section{Processable 2D materials beyond graphene: $\mathrm{MoS}_{2}$ liquid crystals and fibres}

Received 00th January 20xx, Accepted 00th January 20xx

DOI: $10.1039 / \times 0 \times x 00000 x$
Rouhollah Jalili ${ }^{* a}$, Sima Aminorroaya-Yamini ${ }^{* b}$, Tania M. Benedetti ${ }^{a}$, Seyed Hamed Aboutalebi ${ }^{c}$, Yunfeng Chao ${ }^{a}$, Gordon G. Wallace ${ }^{a}$, and David L. Officer ${ }^{a}$

www.rsc.org/

Herein, we show properly engineered $\mathrm{MoS}_{2}$ crystals can readily form liquid crystalline dispersions in water making them ideal candidates for large-scale manufacturing processes. The guideline provided here can serve as the basis to develop practical protocols to address the long-standing goal of large-scale manufacturing of 2D materials.

Two-dimensional transition metal dichalcogenides (2D TMDs), of which $\mathrm{MoS}_{2}$ is the most studied, are the silicon-like 'semiconductor' analogues of graphene. Like graphene, they offer opportunities to develop devices that have no analogue in silicon-based electronics due to their unique band structures, material variability and flexibility. Their versatile electronic, optical, and electrochemical properties offer a new technological potential landscape for the production of transistors, ${ }^{1,} \quad 2$ optoelectronics, ${ }^{3}$ electrocatalysis, ${ }^{4}$ supercapacitors, $^{5}$ batteries $^{6}$ and solar cells. ${ }^{7}$ However, integration of 2D TMDs into macrostructures while preserving their inherent properties requires processable materials that can be directly fabricated using scalable material processing and fabrication approaches without the aid of any processing additives. ${ }^{8-10}$ While significant progress has been made on the production of various 2D TMDs including $\mathrm{MoS}_{2}$ by chemical vapor deposition (CVD) growth, $^{11}$ chemical exfoliation, ${ }^{12}$ electrochemical exfoliation, ${ }^{13}$ and exfoliation in solvents, ${ }^{14}$ the development of a processable 2D TMD dispersion, which is suitable for large-scale manufacturing and processing, has yet to be addressed.

Such a development has indeed occurred for graphene oxide (GO) with the formation of liquid crystalline (LC) dispersions of $\mathrm{GO}^{15-17}$ that have allowed the fabrication of graphene-based

\footnotetext{
Intelligent Polymer Research Institute, ARC Centre of Excellence for Electromaterials Science, AlIM Faculty, Innovation Campus, University of Wollongong, North Wollongong, NSW 2522 (Australia)

${ }^{b}$. Institute for Superconducting and Electronic Materials, AlIM Faculty, Innovation Campus, University of Wollongong, North Wollongong, NSW 2522 (Australia)

Condensed Matter National Laboratory, Institute for Research in Fundamental Sciences (IPM), Tehran 19395-5531, Iran

Electronic Supplementary Information (ESI) available: [Experimental, Figure S1 and S2]. See DOI: $10.1039 / x 0 x x 00000 x$
}

devices using wet-spinning, ${ }^{8,9,18-20}$ printing and coating, ${ }^{21}$ for a broad range of applications in energy storage, ${ }^{9,}$ 22-24 superconductors, ${ }^{25}, 26$ optoelectronics ${ }^{27}, 28$ and structural materials. ${ }^{15,} 29$ In an analogous fashion, LC 2D TMDs could serve as a platform for the rational design and fabrication of TMD-based materials for harnessing and translating their useful properties into practical device applications.

To address this challenge, we introduce processable LC $\mathrm{MoS}_{2}$ nanosheet dispersions (as a model 2D TMD dispersion) to enable large scale fabrication of 3D architectures. We demonstrate that, in contrast to naturally occurring crystals, properly engineered $\mathrm{MoS}_{2}$ crystals can be readily exfoliated into ultra-large $\mathrm{MoS}_{2}$ nanosheets that form LC dispersions in water. We furthermore show that such anisotropic nematic ordering can be exploited to achieve robust $\mathrm{MoS}_{2}$ fibre architectures using wet-spinning.

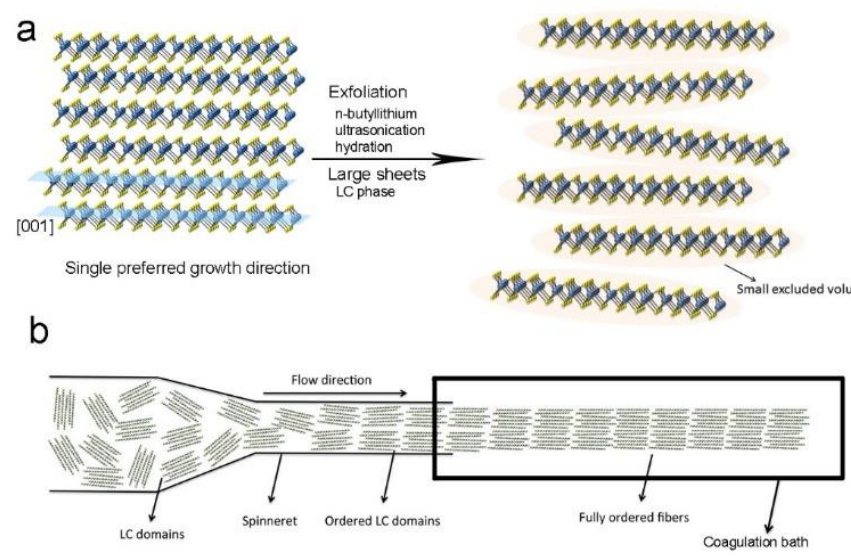

Figure 1. Graphical illustration of the preparation of spinnable 2D MoS 2 nanosheets. (a) A $\mathrm{MoS}_{2}$ crystal with preferential [000I] direction could be easily exfoliated into single large sheets by a standard lithium intercalation and exfoliation using an ultrasonication/hydration-assisted process; this produces a nematic phase at high enough concentration to orient the sheets parallel to each other and thus minimize the excluded volume. (b) If introduced into a suitable coagulation bath, the $\operatorname{MoS}_{2}$ LC domains can be ordered upon applying flow induced shear stress with subsequent alignment and orientation resulting in ordered fibre architecture. 

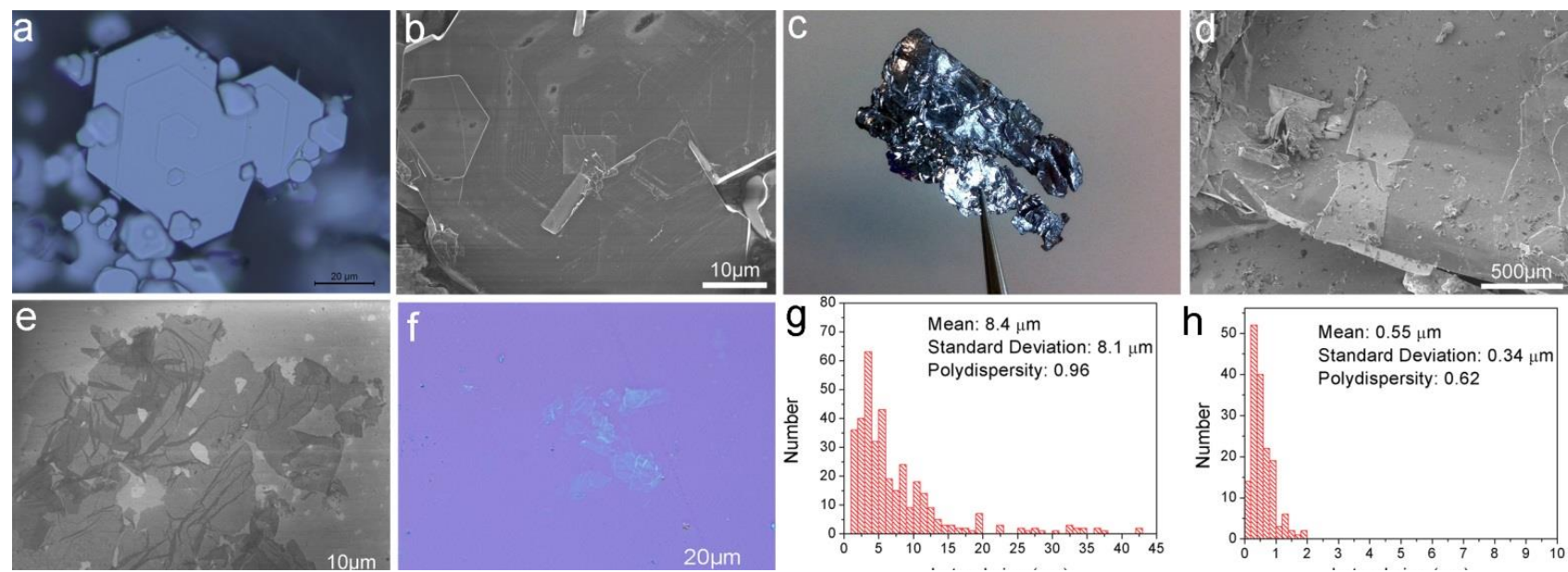

Figure 2. a) Optical and b) SEM micrographs of as-synthesized $\mathrm{MoS}_{2}$ crystals, which show the hexagonal shape of the as-prepared large sheets grown along the basal plane leading to the fabrication large grain crystals with preferred order along the c-axis direction [000I]. c) Optical and d) SEM micrographs of natural MoS ${ }_{2}$ crystal. e) Representative SEM micrograph showing the large sheet size and flexible and transparent nature of the sheets from as-synthesized MoS $\mathrm{Crystals}_{2}$ characteristics also apparent in the optical micrograph presented in (f). It shou ofld be noted that the ability to locate large sheets of $\mathrm{MoS}_{2}$ on a silicon wafer is very valuable as it can provide opportunities to fabricate MoS ${ }_{2}$ based transistors. g) The size distribution of $2 \mathrm{D}$ sheets from as-synthesized $\mathrm{MoS}_{2}$ crystals illustrates the existence of predominantly micrometer-sized sheets that are essential for ensuring a transition from the isotropic phase to the biphasic region, and subsequent nematic phase as seen at $0.5 \mathrm{mg} \mathrm{ml}^{-1}$. h) Size distribution of $2 \mathrm{D}$ sheets after exfoliation of the natural crystal showing the existence of predominantly submicron-sized lateral sizes.

Both rigid and flexible anisotropic molecules undergo a transition from the isotropic phase to the so-called nematic phase as a function of aspect ratio and concentration. ${ }^{16}$ For that, a high temperature solid state reaction ${ }^{30}$ was used to fabricate large grain $\mathrm{MoS}_{2}$ crystals with preferred orientation along the [00OI] direction. This preferred directional growth with weak van der Waals interplanar bonds results in the exfoliation of large $\mathrm{MoS}_{2}$ sheets and subsequent LC formation (Figure 1a).

The as-synthesized crystalline material shows large crystals (more than $10 \mu \mathrm{m}$ ) with screw dislocations in the form of hexagons on the surface due to a non-centrosymmetric arrangement of adjacent layers (Figure $2 a$ and b). ${ }^{31}$ In comparison and as a control material that was also exfoliated, large natural single crystals of $\mathrm{MoS}_{2}$ (Manchester Nanomaterials) show a metallic surface finish with a flake size in the order of a few centimeters with no dislocations (Figure $2 c$ and $d$ ).

Exfoliation was achieved by employing an organolithium compound as a lithiation agent to expand the lattice and obtain an intercalated compound, subsequently exfoliating it into $2 \mathrm{D}$ sheets by an ultrasound-assisted hydration process. ${ }^{32}$ After centrifugation at $11 \mathrm{krpm}$ for $15 \mathrm{~min}$ to remove any nonexfoliated particles, the resulting concentration of the $\mathrm{MoS}_{2}$ dispersion was $0.5 \mathrm{mg} \mathrm{ml}^{-1}$. Scanning electron microscopy (SEM) examination of the as-deposited $\mathrm{MoS}_{2}$ nanosheets on a silicon wafer showed the presence of ultra-large sheets with lateral sizes predominantly larger than 10 micrometers (Figure 2e). The transparent nature of the as-produced $2 \mathrm{D}$ sheets hints at the presence of mostly monolayer $\mathrm{MoS}_{2}$. The pale, transparent and very low optical contrast difference between the substrate and sheets in the micrograph presented in Figure $2 f$ further supports the monolayer nature of the as-exfoliated sheets. The AFM analysis confirmed this observation (Figure S1).

A SEM survey of $250 \mathrm{MoS}_{2}$ sheets demonstrated that the majority of the sheet sizes are in the range of 0.5-20 $\mu \mathrm{m}$ with a mean size of $8.4 \mu \mathrm{m}$, and the presence of sheet sizes as large as $\sim 45 \mu \mathrm{m}$ in the dispersion (Figure $2 \mathrm{~g}$ ). In contrast, the exfoliation of the natural $\mathrm{MoS}_{2}$ crystal gave predominantly submicron flake sizes (Figure $2 \mathrm{~h}$ ), which is in agreement with previous reports in which organolithium chemistry was used for exfoliation. $^{12,33,34}$

As the same exfoliation process was employed for both samples, the unique formation of large sheets from the assynthesized crystals must be a result of its crystalline structure. Figure 3a compares X-ray diffraction (XRD) patterns obtained from both $\mathrm{MoS}_{2}$ materials. Both as-synthesized and natural $\mathrm{MoS}_{2}$ crystals demonstrate large grains with preferred growth orientation along the [000l] crystallographic direction. Therefore, as illustrated in Figure 1a, use of the large $\mathrm{MoS}_{2}$ crystals with preferential growth along the [000I] direction appears to be an essential requirement for effective exfoliation to large sheets and subsequent formation of the LC phase.

In order to explore the difference between the as-synthesized and natural $\mathrm{MoS}_{2}$ crystals, the vibrational properties of both materials were examined by Raman spectroscopy in the range of $360-420 \mathrm{~cm}^{-1}$ (Figure $3 \mathrm{~b}$ ). There are two prominent peaks in the spectra, marked as $E_{2 g}$ and $A_{1 g}$, corresponding to in-plane and out-of-plane vibrational modes, respectively. The lower intensity of the $E_{2 g}$ peak and red shift of the $A_{1 g}$ peak in the assynthesized $\mathrm{MoS}_{2}$ crystal suggest weaker van der Waals coupling of the stacked layers in contrast to that of the natural crystal. $^{31,35,36}$ 

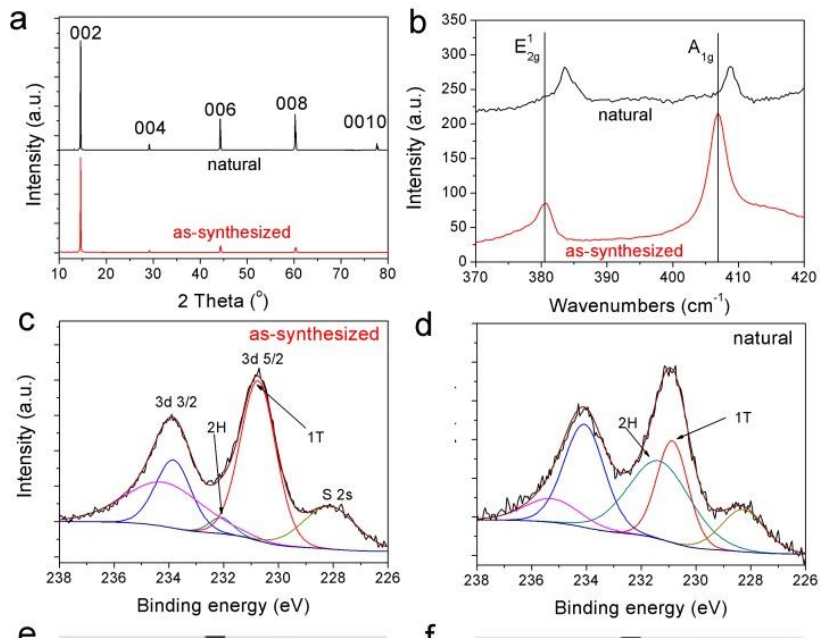

e

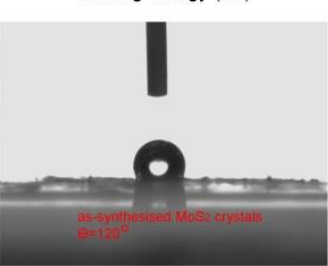

f

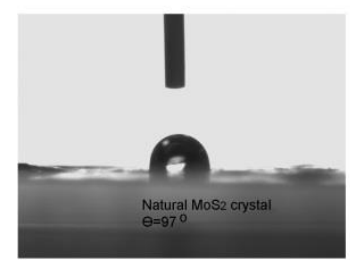

Figure 3. Structural characterization of $\mathrm{MoS}_{2}$ materials. a) XRD patterns of $\mathrm{MoS}_{2}$ crystals showing preferred growth in the c-axis direction [000I]. b) Raman spectra of the $\mathrm{MoS}_{2}$ crystals. In contrast to the natural crystal, the as-synthesized $\mathrm{MoS}_{2}$ crystals show stronger out-of-plane vibrations of $S$ atoms in the opposite direction perpendicular to the (001) plane, also known as the $A_{1 g}$ mode, as well as a shift to lower wavenumber of both the out-of-plane $\left(A_{1 g}\right)$ and in-plane vibrations $\left(E_{2 g}\right)$, indicative of a weaker van der Waals coupling of the stacked layers. The as-synthesized $\mathrm{MoS}_{2}$ crystals exhibited a more metallic structure compared to the natural crystal as evidenced by the comparative XPS spectra (c, d) resulting in a more hydrophobic surface (e) in contrast to the natural crystal (f), leading to a more efficient lithium intercalation. It should also be noted that the $1 \mathrm{~T}$ structure exhibits a meta-stable structure with lower binding energy compared to the $2 \mathrm{H}$ phase, which can facilitate the exfoliation process.

The XPS results (Figure 3c,d) also verified the existence of a much higher amount of a metallic $1 T$ phase compared to a semiconducting $2 \mathrm{H}$ phase in the as-synthesized crystal $(1 \mathrm{~T} / 2 \mathrm{H}$ ratio of 2.12 for the as-synthesized crystal in contrast to 1.2 for the natural crystal). Bearing in mind that the $1 \mathrm{~T}$ phase exhibits a lower binding energy, it is easier for the 1T phase to take part in the lithium intercalation process and consequently be exfoliated more efficiently. Measuring the contact angle of water on both crystals (Figure $3 e, f$ ) demonstrated that the assynthesized $\mathrm{MoS}_{2}$ crystals $\left(\theta=120^{\circ}\right)$ are more hydrophobic than the natural ones $\left(\theta=97^{\circ}\right)$. This in essence means that the as-synthesized crystal has stronger interactions with the reaction solvent (hexane) resulting in a more effective exfoliation process.

Figures $4 \mathrm{a}$, b show cross-polarized optical microscopy (POM) images of the $\mathrm{LC} \mathrm{MoS}_{2}$ dispersion at concentrations of 0.5 and $2.5 \mathrm{mg} \mathrm{ml}^{-1}$, respectively. The existence of predominantly micrometer-sized sheets resulted in a transition from the isotropic phase to the biphasic region, and the subsequent nematic phase as seen as birefringence. Moreover, it should be noted that $\mathrm{MoS}_{2}$ sheets exhibit a highly charged surface profile, and can only approach each other to a defined separation distance rendering the system prone to an entropic-driven configuration of nematic LC phases. The critical theoretical concentration ( $\Phi)$ for the transition between isotropic to nematic phase based on the $\mathrm{MoS}_{2}$ sheets sizes can be calculated based on Equation 1, which serves as a model system for liquid crystal phases of charged 2D platelets. ${ }^{37}$

$\Phi=\frac{3}{8} \sqrt{3} \frac{\mathrm{L}}{\mathrm{D}} \frac{1+\sigma^{2}}{1+3 \sigma^{2}} \rho \mathrm{D}^{3}$

$D^{3}$ is a dimensionless number density for the isotropic to biphasic and, subsequently, biphasic to nematic transition concentrations. L, D, $\sigma$ and $\rho$ are the sheet thickness, the average $\mathrm{MoS}_{2}$ lateral size, polydispersity and the density, respectively. ${ }^{37}$ In the case of $\mathrm{LC} \mathrm{MoS}_{2}$ nanosheets, the calculated transition concentration range is from 0.23 to 0.37 $\mathrm{mg} \mathrm{ml}$. Concentration values below this range indicate isotropic behavior, within the range biphasic behavior and above the range a nematic phase. The obtained concentration for the LC MoS 2 nanosheets $\left(0.5 \mathrm{mg} \mathrm{ml}^{-1}\right)$ falls in the nematic range and thus supports the formation of the LC phase. In contrast, the calculated concentration of the natural $\mathrm{MoS}_{2}$ nanosheets for the formation of the nematic phase $(>13.8 \mathrm{mg}$ $\mathrm{ml}^{-1}$ ) largely exceeded the obtained concentration $\left(\sim 0.3 \mathrm{mg} \mathrm{ml}^{-}\right.$ $\left.{ }^{1}\right)$. The required high concentrations for such small nanosheets are unachievable due to the agglomeration of the sheets.

In the case of $\mathrm{LC} \mathrm{MoS}_{2}$, the high aspect ratio arising from the use of large sheets introduces significant systematic anisotropy into the system resulting in an excluded volume-induced entropic rearrangement. In order to minimize the free energy of such a polydisperse system, the excluded volume of the large sheets limits the free rotation of much smaller sheets and in essence, semi-freezes their orientation, promoting nematic phase formation at low concentrations. When this LC system was subjected to a flow-field, flow-induced optical birefringence was observed (Figure 4c).

To further illustrate this ordering effect, a rheological investigation was carried out on the systems (Figure $4 \mathrm{~d}-\mathrm{f}$ ). A simple shear stress and viscosity vs shear rate curve showed the existence of a clear distinct yield value. This yield value indicates that there is a local minima in energy state, which needs to be overcome before flow can occur. As demonstrated in our previous work on $\mathrm{LCGO}^{21,} 37$ the energy minima reported here could be a result of crowding or jamming. The development of a finite yield stress at such low concentrations also suggests the existence of a weak network structure that breaks down before the viscous flow is induced. ${ }^{21}$ This demonstrates that even at this low concentration, the $\mathrm{MoS}_{2}$ dispersion shows a typical non-Newtonian fluid behavior similar to that of LCGO dispersions. ${ }^{21}$ The descending curve, however, shows much lower stress values and viscosities compared to the first ascending curve suggesting that the orientation of random mesogens has already taken place. The drop in the yield value verifies the alignment of the sample upon introducing a strong enough shear field. 


\section{Journal Name}

\section{COMMUNICATION}
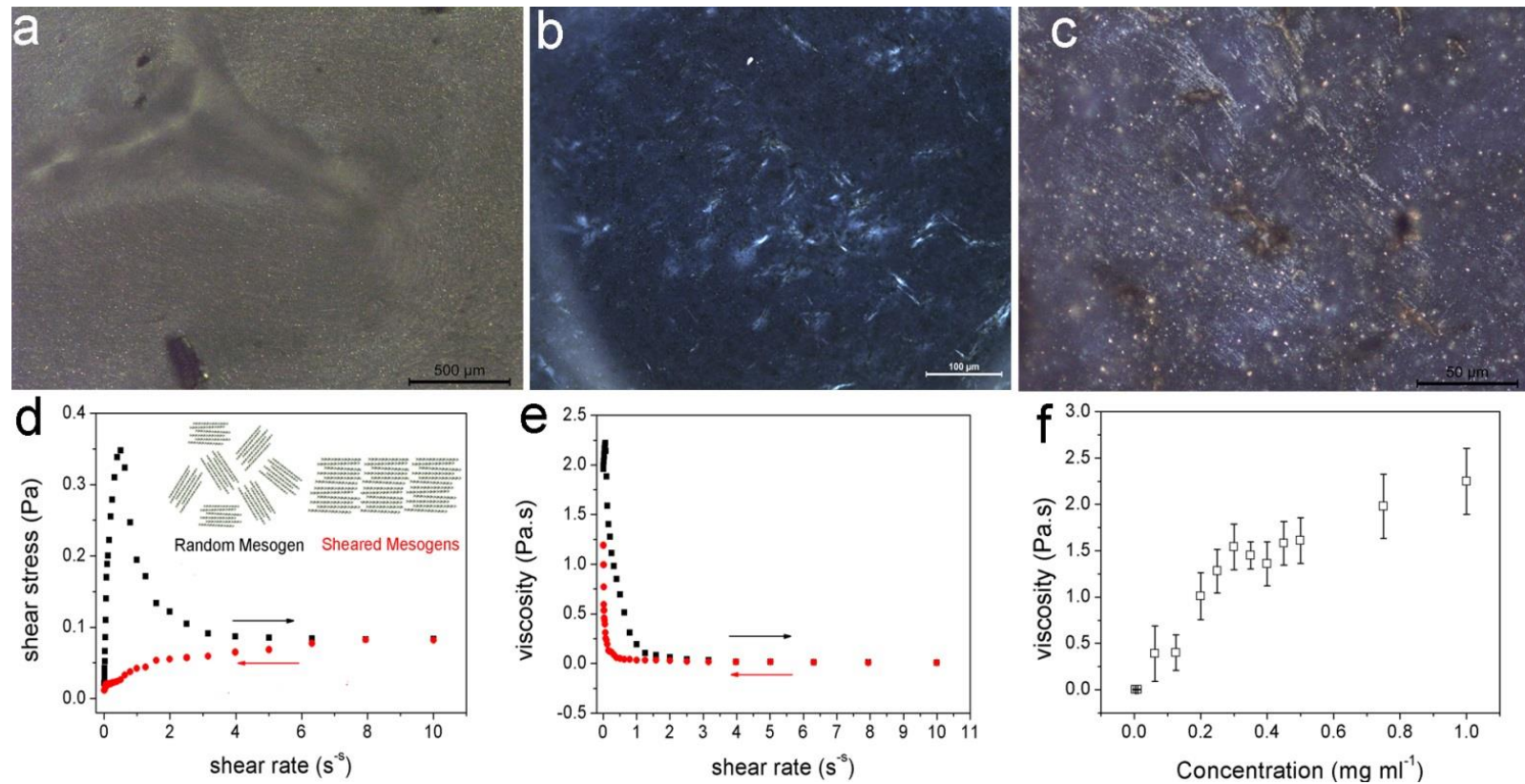

Figure 4. a, b) Cross polarized optical microscopy (POM) images from the $\mathrm{MoS}_{2}$ dispersion showing Schlieren texture in nematic LC MoS 2 at concentrations of 0.5 mg ml ${ }^{-1}$ and 2.5 $\mathrm{mg} \mathrm{ml}^{-1}$, respectively. (c) POM image of the $\mathrm{LC} \mathrm{MoS}_{2}$ dispersion after applying shear using a glass pipette. Upon shear, the nematic texture becomes more pronounced and ordered. d) Shear stress and e) viscosity vs. shear rate of the $0.5 \mathrm{mg} \mathrm{ml}^{-1} \mathrm{LC} \mathrm{MoS}$ dispersion showing two complete ascending-descending flow curves (arrows represent the direction of flow curve measurement). Upon applying shear, the randomly ordered mesogens are sheared and ordered parallel to the direction of the applied shear. However, in order to do so, the system needs to overcome an energy barrier known as the yield value (the lowest shear stress required for viscous flow), which corresponds to the strength of the weak network formed at this concentration. After overcoming this barrier, the dispersion can flow. However, in the descending curve (decreasing shear rate), the yield value drops confirming the orientation and alignment of the anisotropic phase under shear. f) Viscosity as a function of concentration.

The viscosity measurement (Figure $4 \mathrm{f}$ ) shows an increase in the viscosity with $\mathrm{MoS}_{2}$ concentration until a peak is reached at $\sim 0.3 \mathrm{mg} \mathrm{ml}^{-1}$, then a sudden drop is observed. Upon becoming concentrated, the isotropic fluid of two dimensional disk-like $\mathrm{MoS}_{2}$ sheets must undergo a transition to a nematic phase in which the $\mathrm{MoS}_{2}$ sheets adopt a preferred orientation. This ordering results in an increase in free volume. This sudden free volume expansion accompanied by ordering in the direction of shear, because of the spontaneous formation of LC domains, results in the sudden drop of viscosity.

Having identified and demonstrated flow-field induced alignment and orientation of $\mathrm{MoS}_{2}$ dispersions, we applied this understanding to the preparation of useful architectures from $\mathrm{MoS}_{2}$ dispersions. Initially, we used both non-LC and LC MoS dispersions to prepare free standing films by vacuum filtration through a cellulose membrane. After drying the $\mathrm{LC} \mathrm{MoS}_{2}$ layer, a freestanding film with a uniform smooth surface finish could be easily removed from the membrane, while the film from the non-LC dispersion cracked extensively and could not be removed (Figure $5 \mathrm{~d}$ ). It should be noted that for the asprepared small sheet sizes of $\mathrm{MoS}_{2}$, there is usually a need to reinforce the architecture with other materials such as 1D SWNTs in order to attain the paper-like architecture. ${ }^{6}$

The SEM micrographs of the as-prepared LC $\mathrm{MoS}_{2}$ film illustrate the highly-aligned layered film structure (Figure 5a-c). The film forming ability of the LC dispersion can be attributed mainly to the large sheet sizes and the resulting rheological properties. Having large sheets provides sufficient interaction between $\mathrm{MoS}_{2}$ layers to maintain the given structure. In addition, we have previously demonstrated for $\mathrm{LCGO}^{21}$ in an isotropic dispersion that the viscosity is the dominant factor governing the fabrication process. This behavior has a direct impact on the processability of the material, as it implies that "short-range" rearrangements of $\mathrm{MoS}_{2}$ sheets in the dispersion occur rapidly and consequently, shape preservation in the final architecture is impossible. ${ }^{8,9}$ In a biphasic or fully nematic LC dispersion, it is the elasticity imparted by the sheets that plays a crucial role in maintaining the structural integrity needed for fluid-phase fabrication techniques such as simple casting, fibre spinning, or inkjet printing. ${ }^{21}$ Moreover, in contrast with fully isotropic dispersions, LCs are known to afford macroscopic materials with higher microstructural order and enhanced properties. ${ }^{22,23,29}$ 

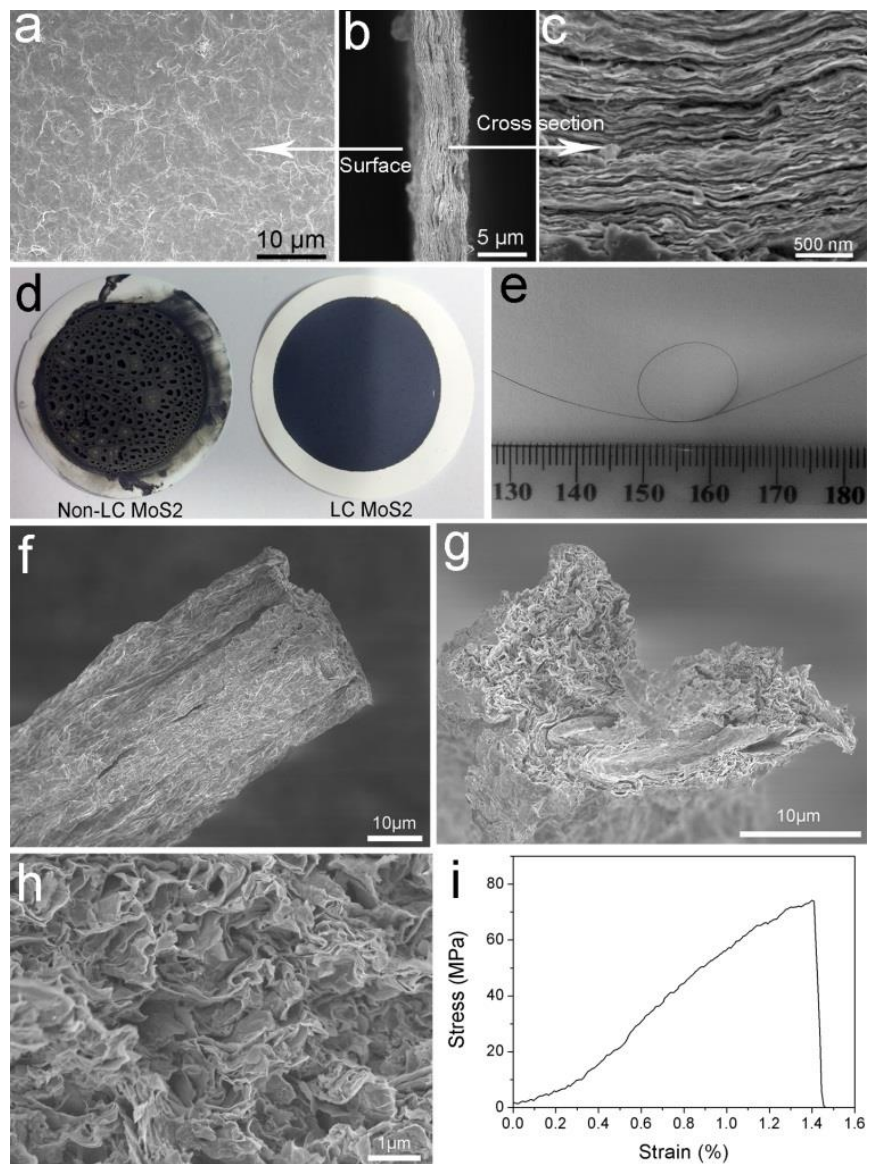

Figure 5. (a-c) Representative SEM micrographs of the as-prepared paper obtained from LC MoS 2 dispersions. The surface of the paper exhibits a continuous but crumbled structure due to a non-uniform drying of the $\mathrm{MoS}_{2}$ large sheets, while the cross-section micrograph shows an aligned architecture as a result of ordered stacking of the $\mathrm{MoS}_{2}$ large sheets. (d) Comparison between paper formation from LC and non-LC MoS dispersion. In contrast to the non-LC dispersions of $\mathrm{MoS}_{2}$, LC dispersions gave a uniform paper-like architecture without cracks. The $\mathrm{LC} \mathrm{MoS}$ dispersions were then used to fabricate fibres $(e, f)$. The representative micrographs show the corrugated surface of a fibre (f) and fibre cross-sections $(g, h)$, which reveal that the sheet planes are aligned parallel to the fibre axis, further verifying the alignment and orientation of the planes along the shear direction during the wet-spinning process. Mechanical tests (i) show the structural integrity of the fibre architecture.

We employed LC $\mathrm{MoS}_{2}$ as the spinning solution for wetspinning to construct macroscopic $\mathrm{MoS}_{2}$ fibres for the first time (Figure 5e). As described in our previous work on graphene fibres, $8,9,37$ the key factor accounting for the fibre spinnability of 2D sheets is the existence of an LC phase. As depicted in Figure $1 \mathrm{~b}$, the liquid crystal mesogens can be oriented over large mono-domains through techniques that impart shear-induced alignment. This offers the opportunity for scalable manufacturing of fibres with structural integrity, without the introduction of a binder that would adversely affect the properties of the free-standing fibres. In a typical wet-spinning procedure, the shear stress applied through the spinneret results in a shear-induced orientation and alignment of the mesogens resulting in a fibrous architecture that can then be rapidly coagulated to preserve the orientation. The fibre exhibits a somewhat wrinkled surface (Figure $5 f$ ). The high magnification SEM micrograph of as-dried fibres reveals a stacked-layered morphology with alignment along the fibre axis (Figure $5 \mathrm{~g}, \mathrm{~h}$ ). The fibres also maintain their structural integrity under stress as revealed by the representative stressstrain curve in Figure $5 \mathrm{i}$. However, it should be noted that these are first generation $\mathrm{MoS}_{2}$ fibres and there is yet ample room to improve the mechanical properties reported here. The metallic nature of the exfoliated $\mathrm{MoS}_{2}$ nanosheets (see XPS data in Figure S1) resulted in high electrical conductivity of the resultant $\mathrm{MoS}_{2}$ fibres (123 S m${ }^{-1}$ ), which opens up a whole range of possibilities to use these fibres as electrodes for supercapacitors, batteries and electrocatalysts.

\section{Conclusions}

In conclusion, the realization of the LC phase in $\mathrm{MoS}_{2}$ dispersions is of significant technological interest, as it paves the way towards the processing of this fascinating material using industrial fabrication techniques such as wet-spinning. In particular, it is envisaged that the method reported here can be used for scalable manufacturing of functional composite inks with different materials (CNTs, metallic particles, polymers, graphene or graphene oxide sheets and other 2D and 1D materials) for use in a wide range of applications. We envision that the concept introduced here can be used to prepare other 2D liquid crystalline materials leading to their practical use beyond electronic applications.

\section{Notes and references}

Funding from the University of Wollongong AlIM for Gold and colbourative grants (RJ \& SAY), Australian Research Council (ARC) Centre of Excellence Scheme (DLO, RG \& GGW, project number: CE 140100012), ARC Discovery Early Career Award (SAY DE130100310), Iran's National Elite Foundation, and Brazilian National Council for Scientific and Technological Development (CNPq-TMB, 229862/2013-6) is gratefully acknowledged. The authors gratefully acknowledge the Materials Node of the Australian National Fabrication Facility (ANFF) for their facilities and research support. The authors acknowledge the use of the UOW Electron Microscopy Centre facilities. Support and guidance from Dr Pawel Wagner is gratefully acknowledged.

1. H. Wang, L. Yu, Y.-H. Lee, Y. Shi, A. Hsu, M. L. Chin, L.-J. Li, M. Dubey, J. Kong and T. Palacios, Nano Lett., 2012, 12, 4674-4680.

2. RadisavljevicB, RadenovicA, BrivioJ, GiacomettiV and KisA, Nat. Nanotechnol., 2011, 6, 147-150.

3. M. S. Choi, D. Qu, D. Lee, X. Liu, K. Watanabe, T. Taniguchi and W. J. Yoo, ACS Nano, 2014, 8, 9332-9340.

4. A. Bruix, H. G. Füchtbauer, A. K. Tuxen, A. S. Walton, M. Andersen, S. Porsgaard, F. Besenbacher, B. Hammer and J. V. Lauritsen, ACS Nano, 2015, 9, 9322-9330. 
5. M. Acerce, D. Voiry and M. Chhowalla, Nat. Nanotechnol., 2015 10, 313-318.

6. J.-Z. Wang, L. Lu, M. Lotya, J. N. Coleman, S.-L. Chou, H.-K. Liu, A. I. Minett and J. Chen, Adv. Energy Mater., 2013, 3, 798-805.

7. M.-L. Tsai, S.-H. Su, J.-K. Chang, D.-S. Tsai, C.-H. Chen, C.-I. Wu, L.-J. Li, L.-J. Chen and J.-H. He, ACS Nano, 2014, 8, 8317-8322.

8. R. Jalili, S. H. Aboutalebi, D. Esrafilzadeh, R. L. Shepherd, J. Chen, S. Aminorroaya-Yamini, K. Konstantinov, A. I. Minett, J. M. Razal and G. G. Wallace, Adv. Funct. Mater., 2013, 23, 5345-5354.

9. S. H. Aboutalebi, R. Jalili, D. Esrafilzadeh, M. Salari, Z. Gholamvand, S. Aminorroaya Yamini, K. Konstantinov, R. L. Shepherd, J. Chen, S. E. Moulton, P. C. Innis, A. I. Minett, J. M. Razal and G. G. Wallace, ACS Nano, 2014, 8, 2456-2466.

10. D. Esrafilzadeh, R. Jalili, E. M. Stewart, S. H. Aboutalebi, J. M. Razal, S. E. Moulton and G. G. Wallace, Adv. Funct. Mater., 2016, 26, 3105-3117.

11. J. Jeon, S. K. Jang, S. M. Jeon, G. Yoo, Y. H. Jang, J.-H. Park and S. Lee, Nanoscale, 2015, 7, 1688-1695.

12. J. Zheng, H. Zhang, S. Dong, Y. Liu, C. Tai Nai, H. Suk Shin, H. Young Jeong, B. Liu and K. Ping Loh, Nat. Commun., 2014, 5.

13. N. Liu, P. Kim, J. H. Kim, J. H. Ye, S. Kim and C. J. Lee, ACS Nano, 2014, 8, 6902-6910.

14. G. Cunningham, M. Lotya, C. S. Cucinotta, S. Sanvito, S. D. Bergin, R. Menzel, M. S. P. Shaffer and J. N. Coleman, ACS Nano, 2012, 6, 3468-3480.

15. R. Jalili, S. H. Aboutalebi, D. Esrafilzadeh, K. Konstantinov, S. E. Moulton, J. M. Razal and G. G. Wallace, ACS Nano, 2013, 7, 39813990.

16. S. H. Aboutalebi, M. M. Gudarzi, Q. B. Zheng and J.-K. Kim, Adv. Funct. Mater., 2011, 21, 2978-2988.

17. Z. Xu and C. Gao, ACS Nano, 2011, 5, 2908-2915.

18. B. Fang, L. Peng, Z. Xu and C. Gao, ACS Nano, 2015, 9, 52145222.

19. Z. Xu, H. Sun, X. Zhao and C. Gao, Adv. Mater., 2013, 25, 188193.

20. Z. Xu and C. Gao, Nat. Commun., 2011, 2, 571.

21. S. Naficy, R. Jalili, S. H. Aboutalebi, R. A. Gorkin lii, K. Konstantinov, P. C. Innis, G. M. Spinks, P. Poulin and G. G. Wallace, Mater. Horiz., 2014, 1, 326-331.

22. S. H. Aboutalebi, S. Aminorroaya-Yamini, I. Nevirkovets, K. Konstantinov and H. K. Liu, Adv. Energy Mater., 2012, 2, 1439-1446.

23. M. M. Islam, S. H. Aboutalebi, D. Cardillo, H. K. Liu, K. Konstantinov and S. X. Dou, ACS Cent. Sci., 2015, 1, 206-216.

24. B. C. Kim, W. J. Cho, W. G. Lee, S. J. Kim, R. Jalili, S. Y. Park, G. G. Wallace, K. H. Yu and S. J. Chang, Synth. Met., 2014, 193, 110-116.
25. K. S. B. De Silva, S. H. Aboutalebi, X. Xu, X. L. Wang, W. X. Li, K. Konstantinov and S. X. Dou, Scr. Mater., 2013, 69, 437-440.

26. M. Mustapić, K. S. B. De Silva, S. H. Aboutalebi, S. Barua, X. Xu, J. Wang, M. S. Hossain, J. Horvat and S. X. Dou, J. Phys. Chem. C, 2015, 119, 10631-10640.

27. T.-Z. Shen, S.-H. Hong and J.-K. Song, Nat. Mater., 2014, 13, 394399.

28. J. Yuan, A. Luna, W. Neri, C. Zakri, T. Schilling, A. Colin and P. Poulin, Nat. Commun., 2015, 6.

29. M. Z. Seyedin, J. M. Razal, P. C. Innis, R. Jalili and G. G. Wallace, Adv. Funct. Mater., 2015, 25, 94-104.

30. M. A. V. Garcia, J. Lindner, A. Sachdev and J. Schwank, J. Catal., 1989, 119, 388-399.

31. L. Zhang, K. Liu, A. B. Wong, J. Kim, X. Hong, C. Liu, T. Cao, S. G. Louie, F. Wang and P. Yang, Nano Lett., 2014, 14, 6418-6423.

32. P. Joensen, R. F. Frindt and S. R. Morrison, Mater. Res. Bull., 1986, 21, 457-461.

33. G. Eda, H. Yamaguchi, D. Voiry, T. Fujita, M. Chen and M. Chhowalla, Nano Lett., 2011, 11, 5111-5116.

34. X. Fan, P. Xu, D. Zhou, Y. Sun, Y. C. Li, M. A. T. Nguyen, M. Terrones and T. E. Mallouk, Nano Lett., 2015, 15, 5956-5960.

35. C. Lee, H. Yan, L. E. Brus, T. F. Heinz, J. Hone and S. Ryu, ACS Nano, 2010, 4, 2695-2700.

36. H. Li, Q. Zhang, C. C. R. Yap, B. K. Tay, T. H. T. Edwin, A. Olivier and D. Baillargeat, Advanced Functional Materials, 2012, 22, 13851390.

37. R. Jalili, S. H. Aboutalebi, D. Esrafilzadeh, K. Konstantinov, J. M. Razal, S. E. Moulton and G. G. Wallace, Mater. Horiz., 2014, 1, $87-$ 91. 